\title{
DISRUPTION MITIGATION WITH HIGH-PRESSURE NOBLE GAS INJECTION
}

\author{
by \\ D.G. WHYTE, T.C. JERNIGAN, D.A. HUMPHREYS, A.W. HYATT, \\ C.J. LASNIER, P.B. PARKS, T.E. EVANS, P.L. TAYLOR, A.G. KELLMAN, \\ D.S. GRAY, and E.M. HOLLMANN
}




\section{DISCLAIMER}

This report was prepared as an account of work sponsored by an agency of the United States Government. Neither the United States Government nor any agency thereof, nor any of their employees, makes any warranty, express or implied, or assumes any legal liability or responsibility for the accuracy, completeness, or usefulness of any information, apparatus, product, or process disclosed, or represents that its use would not infringe privately owned rights. Reference herein to any specific commercial product, process, or service by trade name, trademark, manufacturer, or otherwise, does not necessarily constitute or imply its endorsement, recommendation, or favoring by the United States Government or any agency thereof. The views and opinions of authors expressed herein do not necessarily state or reflect those of the United States Government or any agency thereof. 


\title{
DISRUPTION MITIGATION WITH HIGH-PRESSURE NOBLE GAS INJECTION
}

\author{
by \\ D.G. WHYTE, † T.C. JERNIGAN, \\ C.J. LASNIER, $\diamond$ P.B. PARKS, T.E. EVANS, P.L. TAYLOR, A.G. KELLMAN, \\ D.S. GRAY, ${ }^{\dagger}$ and E.M. HOLLMANN ${ }^{\dagger}$ \\ †University of California, San Diego \\ ¥0ak Ridge National Laboratory \\ $\vartheta_{\text {Lawrence Livermore National Laboratory }}$
}

This is a preprint of a paper presented at the 15th International Conference on Plasma Surface Interactions in Controlled Fusion Devices, May 27-31, 2002, in Gifu, Japan, and to be published in the Proceedings.

\section{Work supported by \\ the U.S. Department of Energy}

under Contracts DE-AC03-99ER54463, W-7405-ENG-48, DE-AC05-000R22725, and Grant DE-FG03-95ER54294 


\begin{abstract}
High-pressure gas jets of neon and argon are used to mitigate the three principal damaging effects of tokamak disruptions: thermal loading of the divertor surfaces, vessel stress from poloidal halo currents and the buildup and loss of relativistic electrons to the wall. The gas jet penetrates as a neutral species through to the central plasma at its sonic velocity. The injected gas atoms increase up to 500 times the total electron inventory in the plasma volume, resulting in a relatively benign radiative dissipation of $>95 \%$ of the plasma stored energy. The rapid cooling and the slow movement of the plasma to the wall reduce poloidal halo currents during the current decay. The thermally collapsed plasma is very cold $(\sim 1-2 \mathrm{eV})$ and the impurity charge distribution can include $>50 \%$ fraction neutral species. If a sufficient quantity of gas is injected, the neutrals inhibit runaway electrons. A physical model of radiative cooling is developed and validated against DIII-D experiments. The model shows that gas jet mitigation, including runaway suppression, extrapolates favorably to burning plasmas where disruption damage will be more severe. Initial results of real-time disruption detection triggering gas jet injection for mitigation are shown.
\end{abstract}




\section{INTRODUCTION}

The avoidance and mitigation of disruptions are critical issues for advancing the tokamak concept as a viable magnetic fusion energy source. A disruption is initiated by some global instability that causes a rapid $\left(\sim 10^{-3} \mathrm{~s}\right)$ thermal quench of the plasma kinetic energy. The resulting resistive plasma causes the confining poloidal magnetic field to decay during the current quench. In-vessel components are damaged by three means: 1) plasma-conducted thermal loading of wall surfaces during the thermal quench, 2) $\mathbf{J} \times \mathbf{B}$ forces from vessel poloidal halo currents during the current quench and 3 ) the conversion of toroidal plasma current into relativistic runaway electrons (RE) that eventually are stopped by the wall.

The magnitude of the damaging effects increase with the plasma kinetic/thermal energy $\left(\mathrm{W}_{\mathrm{th}}\right)$ and magnetic energy $\left(\mathrm{W}_{\mathrm{mag}}\right)$ of a device. While a high-energy density plasma is desired and needed for energy production - it also becomes possible that a single-event uncontrolled disruption will terminate the operational viability of the wall components. Obviously active control of the plasma is needed to avoid conditions that lead to the triggering instability. However, in the event this control fails, it is then desirable to trigger a pre-emptive plasma termination that mitigates simultaneously all of the damaging effects of the oncoming disruption.

We will describe the use of a high pressure jet injection of moderate- $Z$ noble gases to provide disruption damage mitigation. This work is an extension of earlier work with helium gas jets on DIII-D [1]. A physical model of the gas injection and current quench plasma equilibrium is benchmarked against DIII-D data. Based on this model, we further discuss the encouraging extrapolation of the DIII-D results to the more relevant burning plasma regime, since the real concern of disruption damage actually occurs for these devices. For convenience the ITER (Final Design Review , $R=8 \mathrm{~m}, \mathrm{I}>20 \mathrm{MA}$ ) [2] tokamak is used as the example burning plasma experiment. Disruption effects have been examined for this ITER version and found to be severe. 


\section{DESCRIPTION OF EXPERIMENT}

A 70 bar gas reservoir (at room temperature, $300 \mathrm{~K}$ ) equipped with a fastacting solenoid valve (opening time $\sim 1 \mathrm{~ms}$ ) is recessed in a radial port adjacent to the DIII-D plasma (Fig. 1). The valve releases $\sim 4 \times 10^{22}$ particles (atoms or molecules) in $\sim 2-5 \mathrm{~ms}$ into the port which effectively acts as a nozzle for the jet (diameter $=0.15 \mathrm{~m}$, length $\sim 0.5 \mathrm{~m}$ ). We estimate the gas jet neutral density, $\mathrm{n}_{0} \sim 4 \times 10^{24}$ $\mathrm{m}^{-3}$ and ram pressure $\mathrm{P}=\mathrm{n}_{0} \mathrm{~T}=\rho v^{2} \sim 30 \mathrm{kPa}$ on entering the plasma. A pressure gauge at the valve opening confirms that pressures are the same for the different gases injected for these experiment: deuterium $\left(\mathrm{D}_{2}\right)$, helium $(\mathrm{He})$, neon $(\mathrm{Ne})$, and argon (Ar). The effective impurity atom density in the $18 \mathrm{~m}^{3}$ plasma volume is then $\sim 2 \times 10^{21} \mathrm{~m}^{-3}$, or about 70 times the electron inventory of the target plasma.

The target DIII-D lower single-null diverted plasmas used in this study have the following parameters: major/minor radius, $\mathrm{R} / \mathrm{a}$ $=1.7 / 0.6 \mathrm{~m}$, plasma current, $\mathrm{I}_{\mathrm{p}} \sim 1.5 \mathrm{MA}$, toroidal field $\mathrm{B}_{\mathrm{T}}=2.1 \mathrm{~T}$, neutral beam heating power, $\mathrm{P}_{\mathrm{NBI}} \sim 5-7 \mathrm{MW}$, electron density, $\mathrm{n}_{\mathrm{e}}$ $\sim 3 \times 10^{19} \mathrm{~m}^{-3}$, electron temperature $\left\langle\mathrm{T}_{\mathrm{e}}\right\rangle \sim$ $1.5 \mathrm{keV}, \mathrm{T}_{\mathrm{e}, \text { central }} \sim 3 \mathrm{keV}$, electron pressure $<\mathrm{P}_{\mathrm{e}}>\sim 7 \mathrm{kPa}$. Target plasmas have total stored energy of $\mathrm{W} \sim 2.4 \mathrm{MJ}$ with thermal (or kinetic)

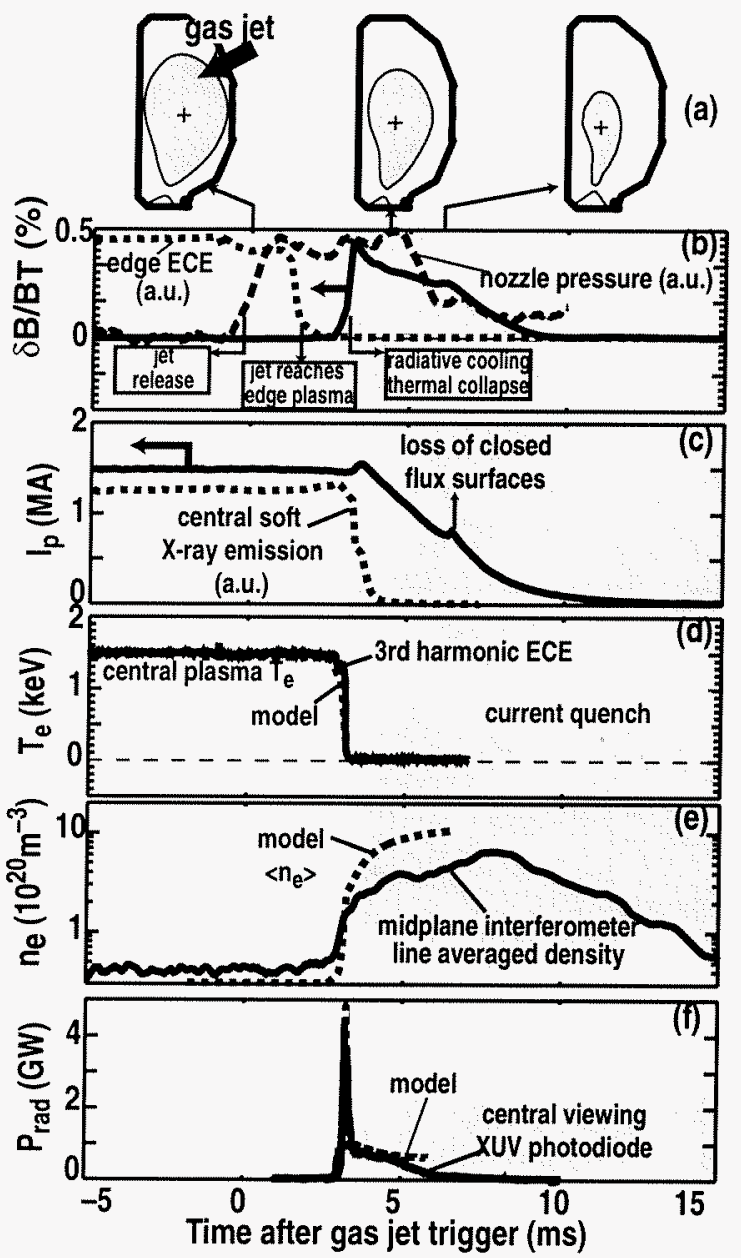

Fig. I. Neon gas jet injection into a stable, neutralbeam heated plasma. (a) Jet geometry and evolution of plasma shape through current quench (shaded region). (b) Jet propagation through the nozzle is indicated by pressure near the valve and density cutoff of edge plasma electron cyclotron emission (ECE). Propagation through the plasma occurs without significant magnetic fluctuations $\left(\delta B / B_{T}\right)$ until the radiative collapse. (c) Plasma current $\left(I_{p}\right)$ and central plasma soft $X$-ray emission. (d) Central plasma electron temperature, $T_{e}$, (e) electron density, $n_{e}$ and $(f)$ radiated power, $P_{\text {rad }}$, are compared to modeling results (see Section 4). energy component, $\mathrm{W}_{\text {th }} \sim 0.6-0.8 \mathrm{MJ}$ and poloidal magnetic energy, $\mathrm{W}_{\text {mag }} \sim 1.6 \mathrm{MJ}$.

In addition to the standard edge and core diagnostic set on DIII-D, several dedicated fast diagnostics are used to study the disruptions. A 30 channel ( $1 \mathrm{MHz}) \mathrm{XUV}$ photodiode radiometer array measures radiated power from the plasma volume. Heat loading of the divertor graphite tiles is measured across the open geometry lower floor $(\mathrm{R}=1-1.8 \mathrm{~m})$ using fast $(20 \mathrm{kHz})$ 
infrared thermography [3]. Halo currents are resolved radially and toroidally by an array of 34 tile current monitors. Central electron temperature is measured using 3rd harmonic (185 GHz, $1 \mathrm{MHz}$ acquisition frequency) electron cyclotron emission (ECE), which does not experience density cutoff for $n_{\mathrm{e}}<2.5 \times 10^{21} \mathrm{~m}^{-3}$. 


\section{MITIGATION OF DISRUPTION DAMAGE}

\subsection{Gas jet injection into stable plasmas}

High-pressure gas jet injection into a stable plasma rapidly dissipates the target plasma energy by radiation and provides a clean rapid quench of the plasma current. An example case of a pre-emptive neon gas jet injection into a stable plasma is shown in Fig. 1. Following the opening trigger to the valve, the jet begins to travel through the port/nozzle as indicated by the fast pressure increase near the valve. The density cutoff of edge 2 nd harmonic ECE indicates the arrival of the jet front at the edge plasma $\sim 1.5 \mathrm{~ms}$ later. After another $1.5 \mathrm{~ms}$ interval, the jet arrives at the central plasma (nozzle length $\sim$ minor radius). Despite the presence of the jet in the plasma, no significant MHD activity $\left(\delta \mathrm{B} / \mathrm{B}_{\mathrm{T}}\right)$ occurs until the very large radiation $\left(\mathrm{P}_{\text {rad }}\right)$ arises from the centrally deposited neon, which collapses the plasma $T_{e}$ and $\beta$ in $\sim 0.2 \mathrm{~ms}$. A rapid exponential current decay is initiated by the thermal collapse and the plasma continues to radiate $\sim 1 \mathrm{GW}$ power. Electron density, $\mathrm{n}_{\mathrm{e}}$, increases by an order of magnitude to $\sim 7 \times 10^{20} \mathrm{~m}^{-3}$ in a few milliseconds. The core plasma remains well centered in the vessel throughout the current quench until closed flux surfaces are lost. Energy balance, which is accurate to within $\sim 100 \mathrm{~kJ}$, indicates that $>95 \%$ of the initial energy of the target plasma is dissipated by radiation.

No collateral damage is caused by the jet injection, either to pumping systems (the vessel pressure is raised to $\sim 10 \mathrm{~Pa}$ by the injection) or other internal systems. Subsequent discharges following the gas jet injection show no indication of radiation from the injected impurity species.

\subsection{Gas jet penetration}

The gas jet is found to penetrate through the plasma at approximately the sonic speed for all gases injected, delivering to the hot central plasma the large quantity of impurity needed for effective disruption mitigation. A cold front, caused by dilution or radiation from deposited gas species, is followed through the plasma using several electron temperature diagnostics (Fig. 2). Electron temperature is a reliable means of following jet penetration since parallel temperature equilibration time $\left(\sim 10^{-6} \mathrm{~s}\right)$ is fast compared to jet penetration time $\left(\sim 10^{-3} \mathrm{~s}\right)$. The jet propagation is consistent both with measured transit velocity of the jet through vacuum and with the expected sound speed (e.g. $\mathrm{c}_{\mathrm{s}} \sim 250 \mathrm{~m} / \mathrm{s}$ for Ar). The various gases also follow closely the expected decrease in sound speed with atomic mass $(\mathrm{m})$, namely $v_{j e t} \propto \mathrm{m}^{-1 / 2}$.

The experimental observations are consistent with the jet penetrating through the plasma as a neutral gas. This is in contrast to low-pressure impurity gas injection using typical fueling valves. In that case the gas is ionized in the plasma periphery and transported in high ion charge states to 
the plasma center on a slower time scale $(\sim 20 \mathrm{~ms})$. The penetration is not consistent with rapid radial plasma transport caused by large radial pressure gradients created by the impurity cooling: Deuterium penetrates equally well as the highly radiating species like neon and argon, and in all cases large magnetic fluctuations do not precede the thermal collapse.

\subsection{Real-time disruption detection and mitigation}

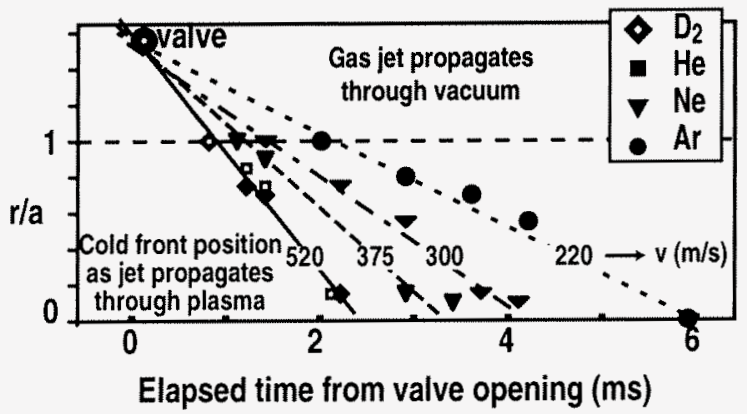

Fig. 2. Jet propagation for different injected gas species through vacuum (in nozzle) and central plasma. The cold front caused by the jet is measured by 2 nd harmonic ECE density cutoff at edge of plasma $(r / a=1)$, Thomson scattering $T_{e}$, soft $X$-ray emission $\left(\propto T^{3 / 2}\right)$ and $3 r$ harmonic ECE in central plasma (no density cutoff for $n_{e}<2.5 \times 10^{21} \mathrm{~m}^{-3}$ ). Effective radial velocities are indicated.

The DIII-D digital plasma control system (PCS) has been used to detect in real-time the onset of a vertical instability caused disruption, typically called a vertical displacement event, VDE (Fig. 3). The PCS triggers the injection of a neon gas jet when the plasma center moves past a variable threshold value set in the PCS for $\Delta Z_{p}$, the vertical displacement of the plasma from it equilibrium position. In these cases, vertical stability is intentionally disabled, during the discharge to initiate the VDE. The time response of the PCS and gas jet is sufficient to terminate rapidly via radiation the plasma before the plasma moves down into the divertor floor. Similar detection algorithms have been developed for disruptions caused by density/radiative limits and tearing modes.

The neon gas jet substantially mitigates the damaging effects at the wall caused by the VDE. Thermal loading of the divertor is minimized by radiative dissipation of $\sim 96 \%$ of the core plasma thermal energy. In contrast, a large portion of $\mathrm{W}_{\text {th }}$ is dissipated in the divertor volume by radiation or by heat conduction to the floor in the non-mitigated case and little radiation occurs from the core plasma in the thermal quench. The reduction of heat conducted to the divertor reduces peak temperatures in the divertor by factors of 2 and 5 at the inner and outer divertor respectively. A summary of divertor conduction heat loading is shown in Fig. 4. The three typical natural (non-mitigated) disruption types find $\sim 50 \%-100 \%$ of the plasma thermal energy directly lost to the divertor. The use of neon or argon gas jet clearly reduces this severe heat load problem, with the energy conducted to the divertor $<10 \% \mathrm{~W}_{\mathrm{th}}$. Peak divertor tile temperatures are typical of those found for a large type-I ELM.

Vessel stresses caused by poloidal halo currents are reduced by a factor of four for these VDE cases (Fig. 3 and Fig. 5). Vessel stress is given by the product of peak poloidal halo current and toroidal peaking factor, both of which are reduced by a factor of two. Of particular 

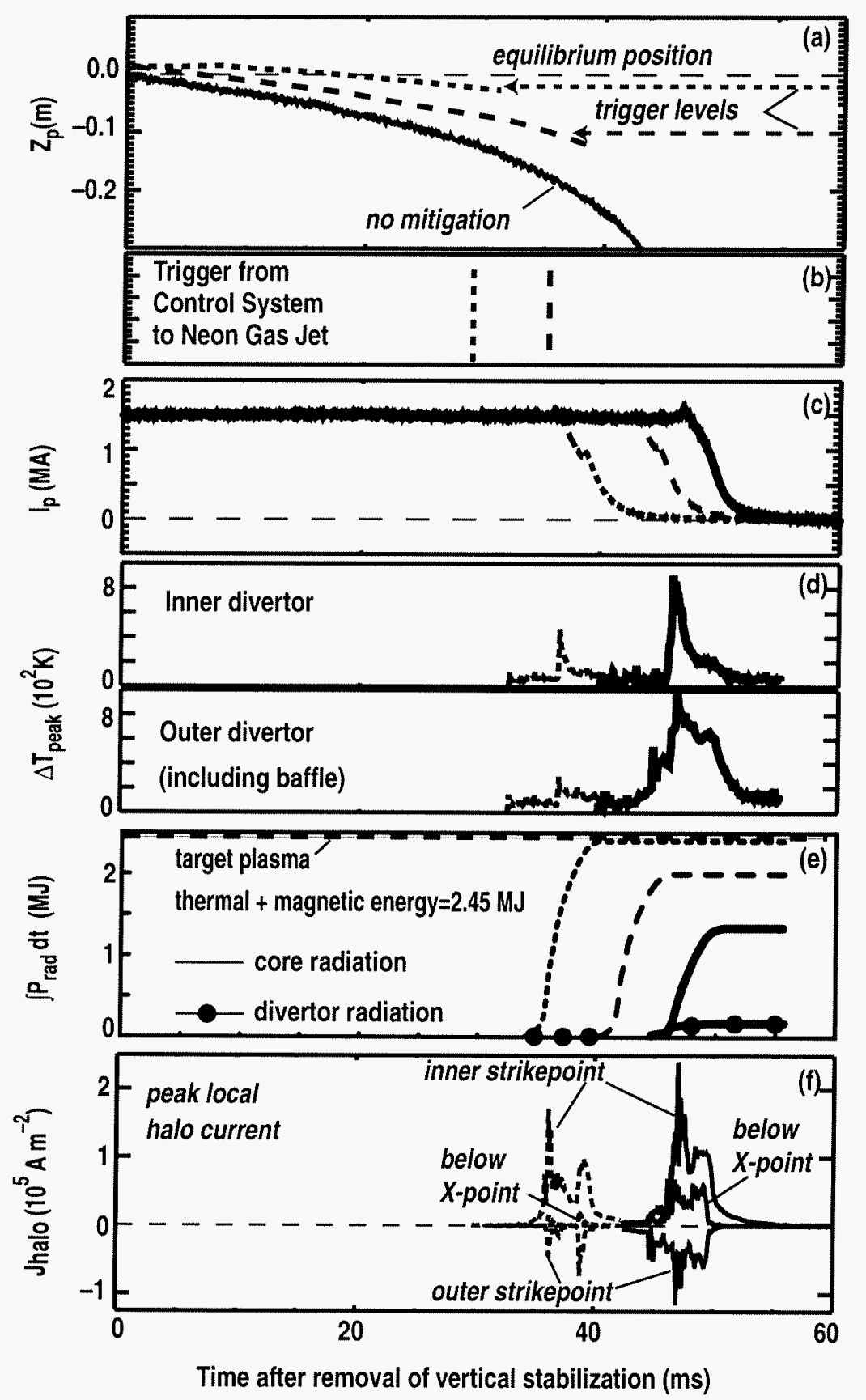

Fig. 3. Real-time detection and mitigation of vertical displacement event (VDE) disruptions. (a) Plasma center vertical position $\left(Z_{p}\right)$ and $\Delta Z_{p}$ trigger levels set in plasma control system. Solid line: no mitigation, dotted line: $\Delta Z_{p}$ $=-2 \mathrm{~cm}$, dashed line: $\Delta Z_{p}=-10 \mathrm{~cm}$. (b) Triggers to gas jet. (c) Plasma current. (d) Reduction of maximum divertor tile temperature rise ( $\Delta T_{\text {peak }}$ ) at inner and outer divertor. (e) Integrated radiated energy from core and divertor plasma. (f) Reduction of maximum divertor halo currents at inner and outer strikepoint positions and directly below the initial $X$-point position.

importance, one finds halo currents essentially eliminated at regions outside the original location of the strikepoints (for example $I_{\text {halo }}$ directly below the $X$-point in Fig. 3). This signifies that we expect reduced stresses on non-target locations in the divertor. Halo currents are reduced because the plasma current decays more rapidly than the plasma movement into the wall. This maintains 
high edge safety factor and minimizes conversion of toroidal current into poloidal halo current. For this reason the mitigation effectiveness is optimized when the gas jet is injected sooner into the vertically unstable plasma (i.e. the $\Delta Z_{\text {threshold }}$ is lower).

\subsection{Control of runaway electrons}

A comparison of the argon pellet injection and argon gas jet illustrates the effectiveness of the gas jet technique in controlling runaway electrons (RE) on DIII-D (Fig. 6). No significant population of RE has yet been found for neon and argon gas jet injection on DIII-D, unlike the pellet which shows a confined RE current $>100 \mathrm{kA}$ in the current quench "tail" and the accompanying non-thermal soft X-ray emission. The two techniques both cause a rapid radiative cooling and current quench. The biggest difference between the techniques is that the gas jet injects $\sim 100$ times the number of atoms into the plasma volume. However, the gas jet creates only modestly higher free electron density increase in the plasma volume $\left(\Delta \mathrm{N}_{\mathrm{e}}\right)$, indicating that the

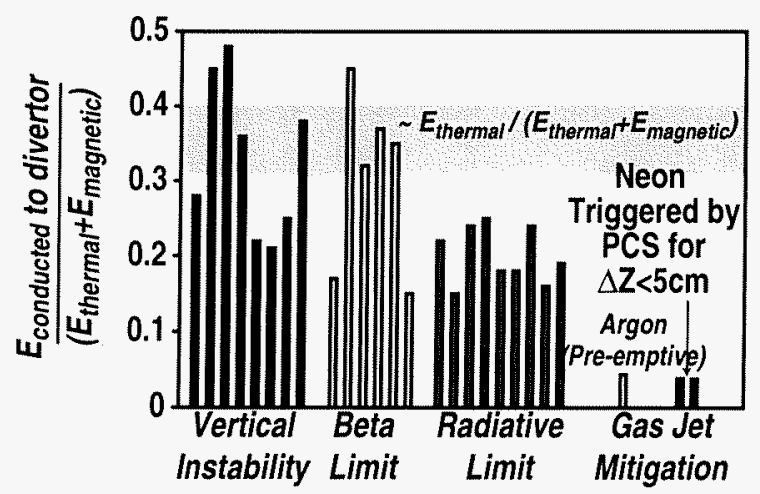

Fig. 4. Summary of divertor thermal load mitigation. The various classes of unmitigated disruptions shows total energy conducted to the divertor $\sim 0.5-1$ of the thermal energy of the target plasma. This is greatly reduced by the use of the neon or argon gas jet injection.

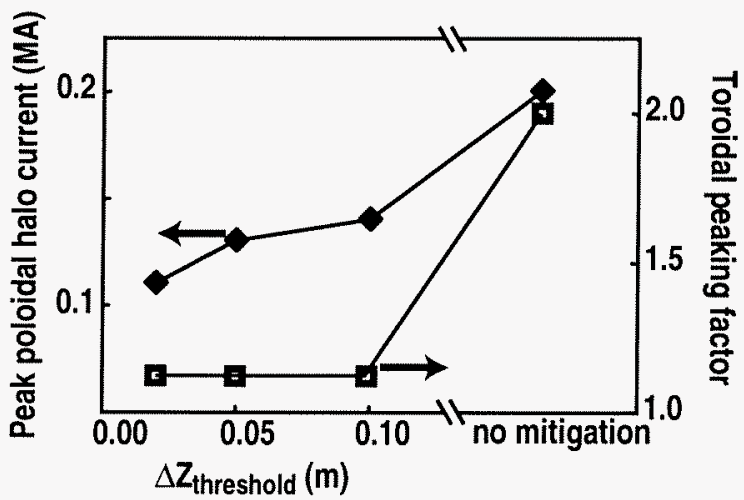

Fig. 5. Summary of halo current mitigation for VDE disruptions versus plasma vertical displacement trigger $\Delta Z_{p}$ for neon jet injection, and for the non mitigated case. The mitigation of peak poloidal halo current and the toroidal peak factor (measured at time of maximum stress to divertor) is optimal for lowest $\Delta Z_{p}$. average charge state of the neon is much lower in the gas jet case. One also sees that the current decay timescale $\left(\tau_{\mathrm{L} / \mathrm{R}}\right)$ is very similar for the two cases, indicating that the accelerating parallel electric field, $E_{\text {par }} \propto 1 / \tau_{\mathrm{L} / \mathrm{R}}$, is similar for the two cases. This is an important feature of the radiative mitigation techniques, particularly with regard to $\mathrm{RE}$ control, that will be addressed in the following section on modeling. 


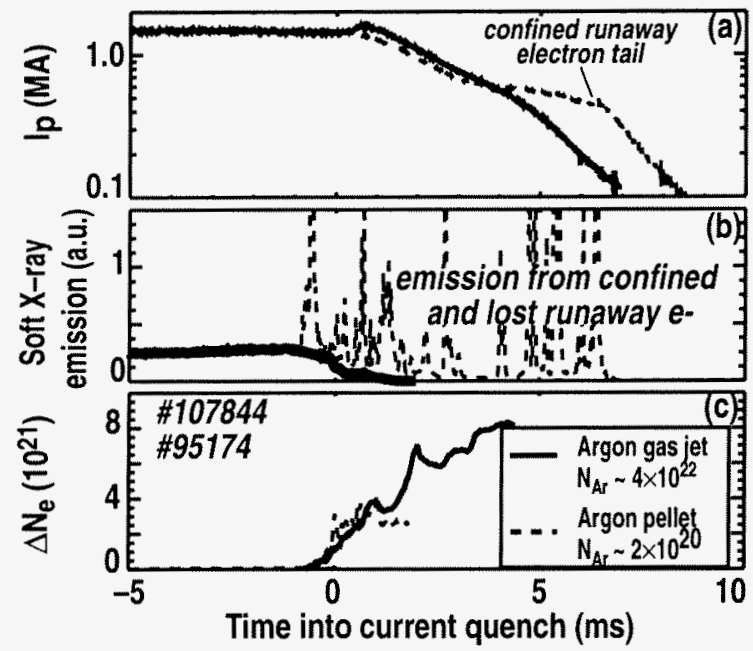

Fig. 6. Demonstration of runaway electron suppression for argon gas jet compared to argon cryogenic pellet injection. (a) Pellet case shows a confined runaway electron tail in plasma current. (b) In pellet case, soft X-ray emissions arise from confined runaways (continuous emission) and loss of runaway electrons to the wall (spikes in emission). (c) Increase in free electron density inventory in plasma $\left(\Delta N_{e}\right)$ indicates gas jet material is weakly ionized compared to pellet case. 


\section{MODELING}

The KPRAD numerical simulation selfconsistently evolves the impurity ionization state distribution and radiation/energy balance for impurity injection mitigation [4]. The code uses charge-state resolved atomic rate coefficients, including radiated power efficiencies $L_{\text {rad }}$ (i.e. coronal equilibrium is not assumed). An example calculation for neon gas jet injection on DIII-D is shown in Fig. 7 and this simulation is compared to DIII-D experimental data in Fig. 1. This simulation uses volume-averaged target plasma parameters (no radial energy or particle transport). The jet species is deposited in plasma at sonic velocity.

One finds that the deposited gas impurity

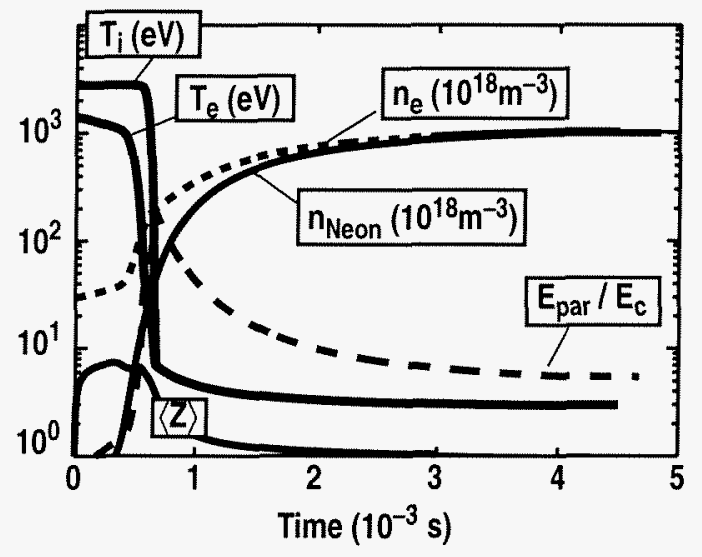

Fig. 7. Example of plasma/impurity thermal and ionization-state evolution from the KPRAD model for neon gas jet injection in DIII-D. The injected neon density (volume averaged from all charge states) $n_{\text {neon }}$, the free electron density $n_{e}$, electron and ion temperature $\left(T_{e}, T_{i}\right)$, the average neon charge state, $\langle Z\rangle$, and the ratio of parallel electric field to critical electric field for runaway production $\left(E_{p a r} / E_{C}\right)$ are shown (units as indicated). Model results of this run are compared to data in Fig. 1.

rapidly quenches the electron plasma kinetic energy through impurity line radiation. The strong electron-ion collisional coupling allows ion energy dissipation, and plasma temperature is decreased from several $\mathrm{keV}$ to $\mathrm{T}_{\mathrm{i}}=\mathrm{T}_{\mathrm{e}} \sim 3 \mathrm{eV}$ in $\sim 0.2 \mathrm{~ms}$, in agreement with data. The initial impurities to reach the plasma are initially burned-through to high charge states. However after the thermal quench further impurities deposited are only singly ionized and strong recombination in the high density plasma eliminates the higher ionization states. In other words, the radiation from the impurities is so overwhelming that the radiative cooling rules out significant further ionization. This effectively clamps the increase in free electron density at $\sim 10^{21} \mathrm{~m}^{-3}$, a key feature of the experimental data.

The parallel current density, $\mathrm{j}\left[\mathrm{A} \mathrm{m}^{-2}\right]$, cannot change on the short time-scale of the thermal quench. Therefore the thermal equilibrium of the current quench plasma $\left(W_{t h} \sim 0, \mathrm{dW}_{\mathrm{th}} / \mathrm{dt} \sim 0\right.$ ) is determined by the equality of impurity-radiated power, $\mathrm{P}_{\mathrm{rad}}\left[\mathrm{W} \mathrm{m}^{-3}\right]$ to ohmic heating, $\mathrm{P}_{\text {ohmic }}$ $\left(=\eta \mathrm{j}^{2}\right)$, namely

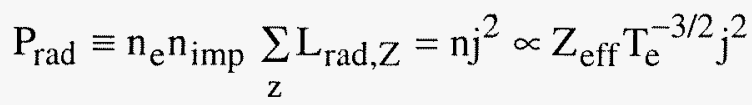


where $n_{e}, n_{i m p}\left[m^{-3}\right]$ are the free electron and impurity densities respectively, $\mathrm{L}_{\mathrm{rad}}\left(\mathrm{Z}_{\mathrm{ii}}\right)\left[\mathrm{W} \mathrm{m} \mathrm{m}^{3}\right]$ is the collisional excitation radiative cooling rate of impurity $Z$ in charge state $i$, and $\eta[\Omega \mathrm{m}]$ is Spitzer resistivity. Eq. (1) determines $T_{e}$ and parallel electric field, $E_{p a r}$ through Ohm's law, namely $E_{p a r}=\eta j$. The current decay $L / R$ time, $\tau_{L / R}$, can then be calculated assuming constant $L$ $=1.5 \mathrm{H}$ for DIII-D case with uniform current distribution.

We validate the model predictions of $\tau_{\mathrm{L} / \mathrm{R}}$ $\propto 1 / \mathrm{E}_{\text {par }}$ and average impurity charge state $\langle\mathrm{Z}\rangle$ against experimental data in Fig. 8 . The model matches well the experimental trends and quantities over the wide range of $n_{i m p}$ found between the pellet and gas jet cases and the different injected species. The most important feature of the model and data is that $E_{p a r}$ remains essentially constant over an extremely large range of $n_{i m p}$ and is sensitive only to the injected species. We can understand this from examining the radiative cooling rate curves for different species (Fig. 9). The equilibrium point between radiated power loss and Joule heating lies well below the ionization potential, I, of each of the species. In this case the functional dependence of the radiated power efficiency is $\mathrm{L}_{\mathrm{rad}} \propto \exp \left(-\mathrm{I} / \mathrm{T}_{\mathrm{e}}\right)$. From Eq. (1) we can then see that $T_{e}$, which determines $\mathrm{E}_{\mathrm{par}}$, depends mostly strongly on I (i.e. species) and only logarithmically with other parameters such as $n_{i m p}, n_{e}$, etc. As $n_{i m p}$ is decreased, higher charge states dominate the radiation (as in the pellet cases). Therefore, while $T_{e}$ increases with the increasing ionization potential of the higher charge states, this effect is mostly cancelled out in the

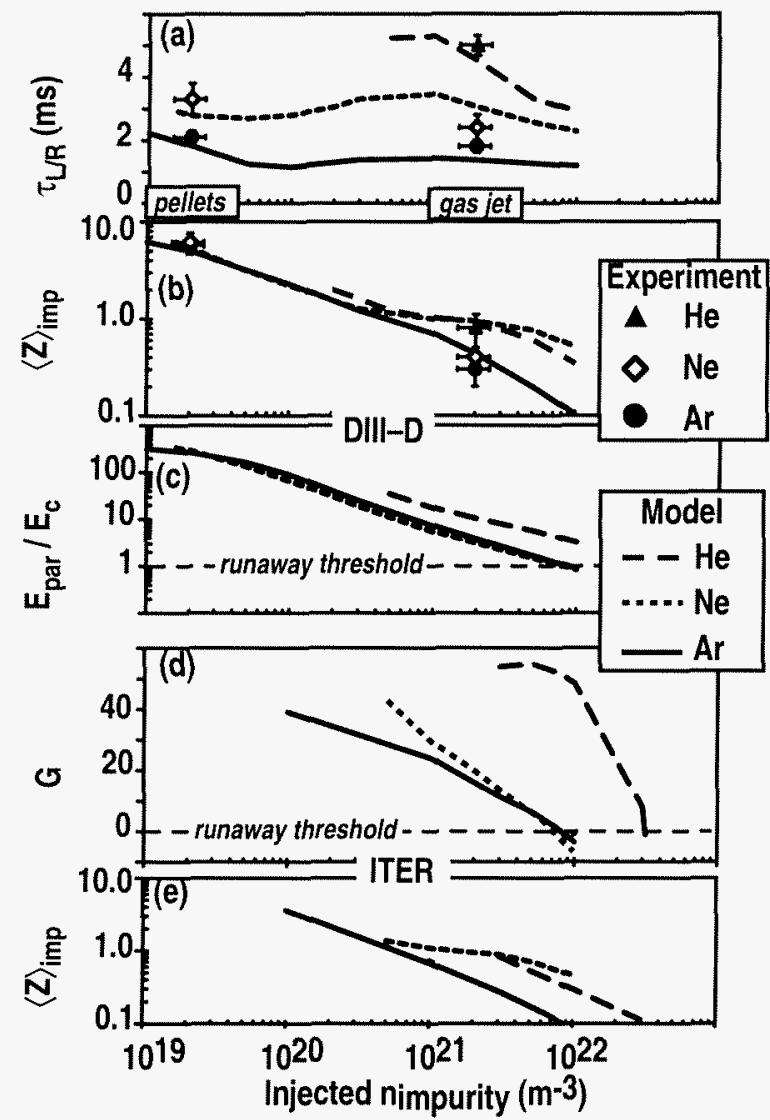

Fig. 8. (a-c) Comparison of KPRAD model to experimental results from DIII-D versus plasma volume averaged density of injected impurity, $n_{\text {imp. }}$ (a) The $L / R$ current decay time $\tau_{L / R^{\propto}} E_{\text {par }}^{-1}$ (b) Average impurity charge state, $\langle Z\rangle_{i m p},(c)$ Ratio of parallel electric field to critical electric field for runaway production $\left(E_{\text {par }} / E_{C}\right)$. (d-e) KPRAD results for ITER with $\langle T\rangle=$ $10.5 \mathrm{keV}, V=1890 \mathrm{~m}^{-3}, j=0.55 \mathrm{MA} \mathrm{m}^{-2}, R, a=8,2.8 \mathrm{~m}$, $L=13 \mu \mathrm{H}$. (d) Runaway gain amplification exponent $(G)$ and (e) average charge state. resistivity term $\left(\propto Z_{\text {eff }} / T^{3 / 2}\right)$ by the increasing $Z_{\text {eff }}$. We note that Spitzer resistivity has previously been verified experimentally in DIII-D during the current quench of a gas jet injection [5]. Therefore, the independent confirmations of $\eta, E_{p a r}$ and $Z$ in Fig. 8 also validate the calculated $\mathrm{T}_{\mathrm{e}}$. 
Relativistic runaway electrons (RE) are produced when $E_{\text {par, which accelerates }}$ electrons, is greater than the critical electric field, $\mathrm{E}_{\mathrm{c}}$, set by collisional drag, namely $\mathrm{E}_{\mathrm{c}}$ $[\mathrm{V} / \mathrm{m}]=\mathrm{mcv} / \mathrm{e} \sim 10^{-21} \mathrm{n}_{\mathrm{e}, \mathrm{T}}$. The definition of $\mathrm{n}_{\mathrm{e}, \mathrm{T}}$ includes both free, $\mathrm{n}_{\mathrm{e}}$, and bound electrons since both contribute to collisional drag of relativistic electrons [6]. RE experience amplification via the knock-on avalanche process in the current quench. Total RE amplification factor is given by, $\mathrm{e}^{\mathrm{G}}$, where the amplification exponent $G=\gamma_{R E} \tau_{L / R} \propto$ $\gamma_{\mathrm{RE}} / \mathrm{E}_{\mathrm{par}}$ and the $\mathrm{RE}$ growth rate $\gamma_{\mathrm{RE}} \propto$ $\left(\mathrm{E}_{\mathrm{par}} / \mathrm{E}_{\mathrm{c}}-1\right)$ [7]. Therefore runaway electrons and their amplification are suppressed when

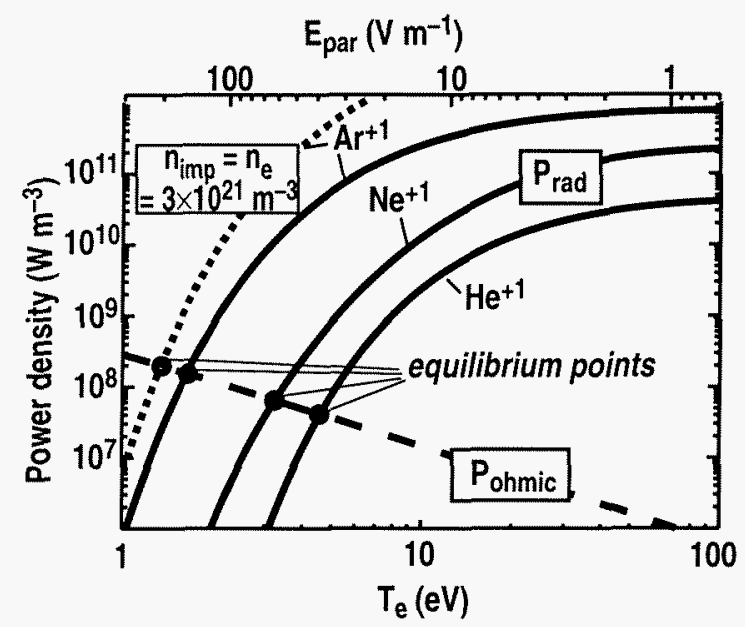

Fig. 9. Current quench $T_{e}$ (or $E_{\text {par }}$ ) is determined by the crossing of ohmic and radiated power densities for singly ionized noble gases. Case shown: $n_{\text {imp }}=n_{e}=$ $10^{21} \mathrm{~m}^{-3}, j=10^{6} \mathrm{~A} \mathrm{~m}^{-2}, Z_{\text {eff }}=1$, except dotted line for $A r^{+1}$ with $n_{\text {imp }}=n_{e}=3 \times 10^{21} \mathrm{~m}^{-3}$ indicating insensitivity of $T_{e}$ to $n_{i m p}$. $\mathrm{E}_{\mathrm{par}} / \mathrm{E}_{\mathrm{c}}<1$ (i.e. the Dreicer evaporation criterion is broken).

The RE amplification gain is calculated in the mitigation scenarios from the benchmarked KPRAD disruption model results $\left(E, \tau_{C Q}\right.$, etc.) and the analytic growth rate formula from [3] for both DIII-D and ITER [Fig. 8(d)]. The model indicates RE suppression $\left(\mathrm{E}_{\mathrm{par}} / \mathrm{E}_{\mathrm{c}}<1\right)$ to occur with $n_{\mathrm{imp}}>7 \times 10^{21} \mathrm{~m}^{-3}$ using gas jets of neon or argon in both devices. This is only a factor of three larger than our current experimental value, $n_{i m p} \sim 2 \times 10^{21} \mathrm{~m}^{-3}$. This result arises primarily from the increasing neutral gas density as $n_{i m p}$ is increased and $\langle Z\rangle$ decreases. Meanwhile, the accelerating $E_{p a r}$ is nearly constant as $n_{i m p}$ increases (as explained above). Therefore, $n_{e}, T$ and $E_{c}$ can in a sense be "arbitrarily" increased by orders of magnitude by the large $n_{\text {imp }}$ from the gas jet. At lower values of $n_{i m p}$ (e.g. pellet injection), we recover the previous result that $R E$ amplification is so large $\left(\mathrm{e}^{40}\right)$ that the majority of plasma current will convert into relativistic electrons. We note that RE are suppressed in DIII-D experiments at a lower value of $n_{\text {imp }}$ than predicted by the model. This is likely a consequence of the finite transport losses of RE, which are not considered in our calculations, and which makes the model a conservative estimate of the necessary $n_{i m p}$ for RE suppression. Neon plus argon are efficient at suppressing RE compared to He because of the larger number of bound electrons per atom. Simultaneously neon and argon provide good thermal mitigation due to their high radiation efficiencies. 


\section{DISCUSSION}

In general, the goal of disruption mitigation is to dissipate the plasma energy by relatively benign isotropic radiation uniformly to the vessel surfaces. Gas jet injection mitigation clearly succeeds in this goal since the radiated power from the injected impurity competes against heat conduction time scale as seen in natural disruptions or ELMs $(\sim 0.5 \mathrm{~ms})$. Importantly, the jet itself does not seem to trigger large MHD instability until the impurity has been deposited in the plasma and allowed to dissipate the energy. The KPRAD model demonstrates that this rapid cooling is also possible in the burning plasma environment, where $\langle\mathrm{T}\rangle \sim 10 \mathrm{keV}$. The rapid cooling and central positioning of the current quench plasma will also reduce halo currents as in the DIII-D case. The question remains that even with this radiative dissipation, is the thermal pulse to the wall sufficient to cause melting or ablation? While the answer to this question will depend on the specific attributes of the device in question (stored energy, surface area, etc.) a previous calculations for ITER [8] showed the beryllium wall surfaces did not melt from a radiative plasma termination using a pellet.

Practical mitigation of DIII-D wall damage caused by disruptions has been demonstrated using the gas jet (Fig. 10). The non-mitigated $\beta$-limit disruption with high stored thermal energy $(\sim 2 \mathrm{MJ})$ is capable of heating graphite divertor tiles and tile edges past the carbon ablation temperature. Previous experiments on DIII-D showed that ATJ graphite produces significant dust particulates ( microns) when overheated [9]. We therefore speculate that the increased breakdown phase carbon radiation in the discharge following the disruption is caused by liberated dust. This radiation and recycling enhancement leads to a loss of control of the current profile $\left(\ell_{\mathrm{i}}\right)$ evolution and as a result the discharge did not meet performance expectations. The increased $\mathrm{C}$ radiation is absent after the gas jet injection, because the wall heat loading is minimized, and good plasma performance was obtained. Also important, there is no indication of enhanced radiation losses from the recycling of the gas jet injected impurity. This benign behavior occurs because the recycling noble gas does not interact chemically with the wall and ion implantation of the impurity into the graphite is minimal due to the low sheath potential $\left(\mathrm{T}_{\mathrm{e}}<\right.$ $2 \mathrm{eV}$ ) in the cooled current quench plasma.

Efficient penetration of gas jet to central plasma seems to play an important role in the effectiveness of mitigation. While a fully developed model on gas jet penetration is not yet available, we hypothesize that the gas penetration is due to the high local neutral pressure and density of the jet. We note first that the DIII-D gas jet has passed an important threshold, namely, the local ram pressure of the jet $(\sim 30 \mathrm{kPa})$ exceeds the volume-averaged plasma electron pressure $(\sim 7 \mathrm{kPa})$. Therefore, in the hydrodynamic sense it is difficult for the plasma to 


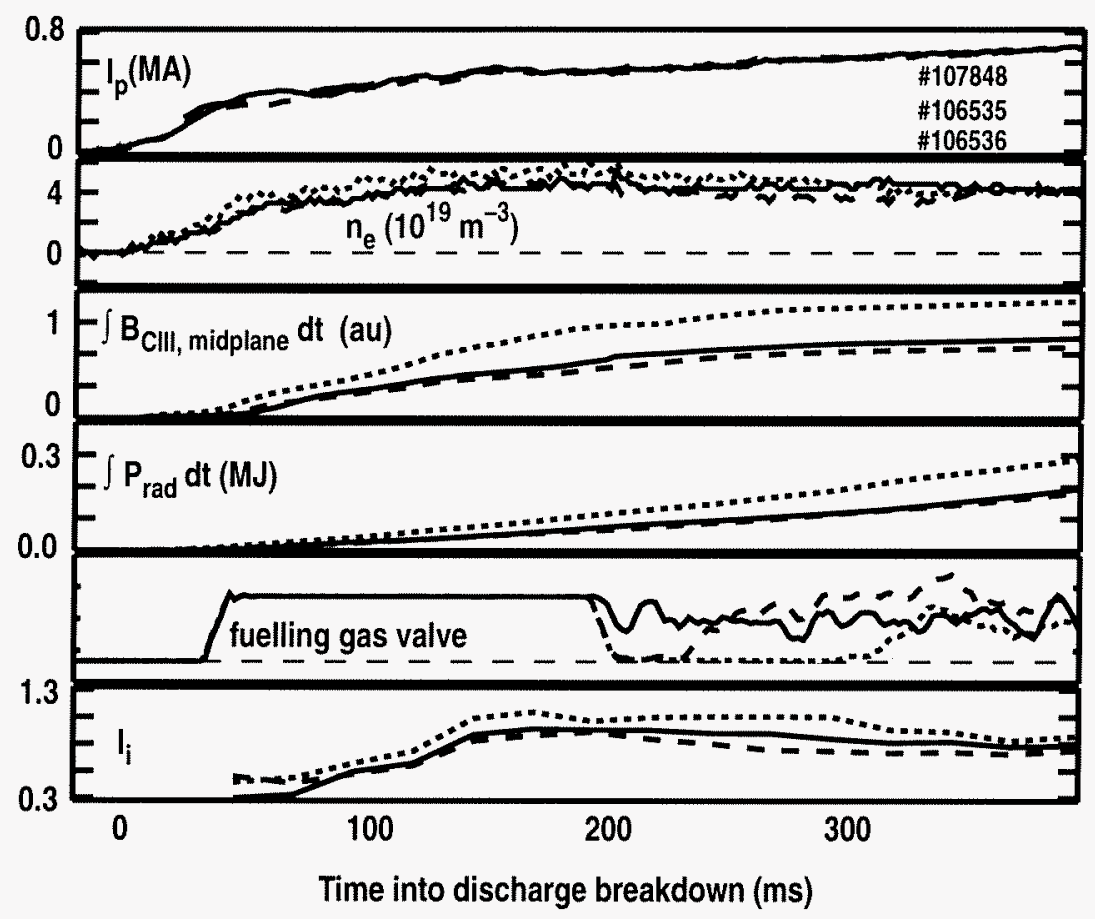

Fig. 10. Discharge breakdown conditions for a reference high performance discharge (solid line) which ended in an unmitigated high- $\beta$ disruption, the subsequent discharge (dotted line) which attempted to repeat the reference but failed, and the successful repeat of the reference after a discharge terminated with argon gas jet mitigation (dashed line). Shown are plasma current $\left(I_{p}\right)$, electron density $\left(n_{e}\right)$, integrated $C^{+2}$ radiation $\left(B_{C} C I I\right)$, integrated total radiated power $\left(P_{\text {rad }}\right), D_{2}$ gas fueling rate, and internal inductance $\left(\ell_{i}\right)$.

stop the forward motion of the jet until it reaches the higher-pressure central plasma $(\sim 40 \mathrm{kPa})$. However, we note that the ablation or heating pressure (as known for pellets [10] exceeds the ram pressure.

Our current data and hypothesis then suggests that the key to jet penetration is that the jet pressure exceeds the plasma pressure. In order for the jet to penetrate to the center of a hotter $\left(\left\langle\mathrm{T}_{\mathrm{e}}\right\rangle \sim 10 \mathrm{keV}\right)$ and higher pressure $\left(\left\langle\mathrm{P}_{\mathrm{e}}\right\rangle>100 \mathrm{kPa}\right)$ burning plasma, a modest increase from our DIII-D jet seems necessary. Restricting the injection cross-section with a specifically designed nozzle (rather than just the vacuum port) and moving the jet valve/reservoir close to the plasma will readily accomplish this. The proximity to the plasma has the further benefit of reducing the time-of-flight for the jet to the central plasma. Since the measured mitigation of thermal loads and halo currents seems linked to the efficient penetration of the jet to the core, it is important that further theoretical and experimental work be carried out on this subject. 


\section{CONCLUSIONS}

High-pressure gas injection of moderate-Z noble gas (neon or argon) reduces simultaneously the three major damage concerns in a tokamak. The problem of divertor thermal loading is mitigated by delivering large quantities of impurity into the core plasma to dissipate $>95 \%$ of the plasma energy by relatively benign, isotropic radiation. Mechanical $\mathrm{J} \times \mathrm{B}$ stresses from poloidal halo currents are reduced because the rapid energy quench provided by the jet leads to a uniform resistive plasma that remains centered in the vessel while toroidal current decays. The large volume density of bound electrons produced by the gas injection can suppress the generation of runaway relativistic electrons in the current quench, which experience a large parallel electric field.

A physical model that describes the thermal and ionization balance of the plasma and gas jet material has been successfully validated against DIII-D experimental data. The model predicts that a gas jet will effectively mitigate disruption damage in burning plasma experiments. The largest uncertainty in the model remains the understanding and extrapolation of the gas jet penetration through the core plasma of a burning plasma device. A simple empirical hypothesis based on jet ram pressure leads us to expect penetration, but further work is clearly needed in this area. 


\section{REFERENCES}

[1] P.L. Taylor, et al., Phys. Plasmas 6, (1999) 1872.

[2] ITER Physics Expert Groups, Nucl. Fusion 39, (1999) 2577.

[3] T.E. Evans, et al. J. Nucl. Mater. 241-243 (1997) 606.

[4] D.G. Whyte, et al., Proc. of 24th European Conference on Controlled Fusion and Plasma Physics, Berchtesgaden, Germany, 1997, Vol. 21A (European Physical Society, 1997) p. 1137.

[5] D.A. Humphreys, D.G. Whyte, Phys. Plasmas 7, (2000) 4057.

[6] P.B. Parks, M.N. Rosenbluth, S.V. Putvinski, Phys. Plasmas 6, (1999) 2523.

[7] M.N. Rosenbluth and S.V. Putvinski, Nucl. Fusion 37, (1997) 1355.

[8] B.V. Kuteev, V. Yu Sergeev, S. Sudo, Nucl. Fusion 35, (1995) 1167.

[9] O.J. Buzhinskij, private communication.

[10] P.B. Parks, M.N. Rosenbluth, S.V. Putvinski, T.E. Evans, Fusion Technology, 35 (1999) 267. 


\section{ACKNOWLEDGMENT}

Work supported by U.S. Department of Energy under Grant DE-FG03-95ER54294, and Contracts DE-AC05-00OR22725, DE-AC03-99ER54463, and W-7405-ENG-48. 